\section{CPS-340 PROFILE OF PATIENTS WITH ABUSIVE CONSUMPTION OF QUICK RELEASE FENTANYL IN A HEALTHCARE AREA}

${ }^{1}$ M Sáez-Torres De Vicente*, ${ }^{2} \mathrm{JA}$ Plata Illescas, ${ }^{3} \mathrm{~T}$ López-Viñau Lopez, ${ }^{3}$ A Perea Pérez. ${ }^{1}$ Hospital Universitario De Badajoz, Pharmacy, Badajoz, Spain; ${ }^{2}$ Hospital Infanta Margarita, Primary Care Pharmacist, Cordoba, Spain; ${ }^{3}$ Hospital Universitario Reina Sofía, Pharmacy, Córdoba, Spain

\subsection{6/ejhpharm-2021-eahpconf.172}

Background and importance In recent years, the prescription of immediate release fentanyl (whose indication is breakthrough pain of oncological origin treated with a basic opioid analgesic) has increased, in many cases for off-label indications, and some patients may develop physical dependence or addiction. Aim and objectives The objective of this study was to describe the type of patients with abusive consumption (those who exceeded the defined daily dose (DDD)) that may have developed dependence to immediate release fentanyl in a healthcare area.

Material and methods This was an observational descriptive study carried out in a healthcare area of 256807 inhabitants, which included all patients who exceeded the DDD for immediate release fentanyl between December 2019 and March 2020. The variables collected were: sex, age, indication for the opioid, duration of treatment, prescribing service, presence of addictions, psychotropic treatment and concomitant analgesic. The data were obtained from the Diraya digital medical records and microstrategy database. Statistical analysis was performed with the $\mathrm{R}$ programme.

Results We detected 32 patients, 58.6\% women, with a median age of 59 (31-87) years, who exceeded an average of 3 (1.1-10) times the immediate release fentanyl DDD, in its different presentations: transmucosal $(n=18)$, sublingual $(n=8)$, lozenge $(n=5)$ and nasal spray $(n=1)$. Mean duration of treatment was 29.93 (2-65) months. In 31\% of cases the indication was oncologic pain, while the rest were for chronic noncancer pain (CNCP): radiculopathy $(n=6)$, osteoarthritis $(n=5)$, low back pain $(n=4)$, phantom limb pain $(n=2)$, peripheral arterial disease $(n=2)$, knee replacement $(n=1)$, polymyalgia rheumatica $(n=1)$ and postradiotherapy laryngeal oedema $(n=1)$. Regarding treatment, only $51.7 \%$ were prescribed fentanyl patch; 52\% were prescribed other opioids (tapentadol $(\mathrm{n}=8)$, oxycodone/naloxone $(\mathrm{n}=3)$, morphine $(\mathrm{n}=2)$, tramadol $(\mathrm{n}=2)$ or paracetamol/codeine $(\mathrm{n}=2)), 86 \%$ NSAIDs and $36 \%$ medications for neuropathic pain (pregabalin, gabapentin or lidocaine patches). $79.3 \%$ of patients were receiving treatment with benzodiazepines, $44.8 \%$ antidepressants and $10 \%$ antipsychotics. In $38 \%$ of cases, the first prescription was in primary care, $31 \%$ in oncology, $24 \%$ in the pain unit and $7 \%$ in cardiovascular surgery. Only one patient had a previous addiction, to parenteral drugs.

Conclusion and relevance In our study, the profile of patients with abusive consumption of rapid release fentanyl was a higher proportion of women, receiving concomitant treatment with benzodiazepines and other analgesics, although with no relation to other addictions or psychiatric pathologies. It should be noted that in most cases it was prescribed off-label (CNCP) and the first prescription was made in primary care.

\section{REFERENCES AND/OR ACKNOWLEDGEMENTS}

Conflict of interest No conflict of interest

\section{CPS-341 THE ROLE OF PHARMACISTS IN PROVIDING PHARMACEUTICAL CARE FOR PATIENTS WITH HEADACHES}

0 Reshetko, A Grishin*. Saratov State Medical University named after VI Razumovskiy of the Health Ministry of the Russian Federation, Department of Pharmacology, Saratov, Russia CIS

\subsection{6/ejhpharm-2021-eahpconf.173}

Background and importance According to statistics from the World Health Organization, at least half of the world's adult population experience headaches at least once a year. Patients suffering from headaches do not always have the opportunity to visit a doctor and therefore an important role in helping these patients is assigned to pharmacists.

Aim and objectives Based on a pharmacoepidemiological study, to identify the features of providing pharmaceutical care to patients with headaches (conversations with the patient, behaviour and knowledge of the pharmacists, priority recommendations and factors affecting them).

Material and methods A pharmacoepidemiological study was conducted, which was based on a survey of 153 pharmacists who completed a questionnaire. The data were processed using Microsoft Excel.

Results Pharmacists noted that they often had requests to recommend a headache drug (66.7\%); most often the request was not defined (56.9\%). More than half of the respondents $(54.9 \%)$ could recommend a prescription drug; $80.4 \%$ would release a prescription drug without a prescription if there was a doctor's instruction or a list of recommendations. The most commonly used groups of recommended drugs were (M01A) non-steroidal anti-inflammatory drugs (92.2\%), analgesics in combinations $(91.5 \%)$ and (N02B) non-narcotic analgesics $(85.0 \%)$. Of the groups recommended for patients, international non-proprietary name (INN) of (M01A) accounted for $45.0 \%$ and INN of (N02) for $55.0 \%$. The most recommended drugs were ibuprofen (62.8\%) and a combination of drotaverin + caffeine + naproxen + paracetamol + phenylephrine (41.2\%). Among the factors influencing the recommendation, the most important were contraindications $(4.2 \pm 0.17)$, proven efficacy and safety $(3.98 \pm 0.19)$ and the patient's age $(3.86 \pm 0.18)$. When communicating with a patient, pharmacists often asked questions related to age $(96.7 \%)$ and previous experience of taking drugs (83.0\%), but rarely questions related to the character and duration of the headache. In most of the simulated situations (pregnancy, child, head injury, old man), pharmacists could advise on some drugs, but the choices were not always rational.

Conclusion and relevance Pharmacist's recommendations were often associated with general factors and questions unrelated to the specifics of the headache. Also, recommendations of combined analgesics indicated an irrational choice of drug. Rational consulting algorithms are needed to improve the knowledge of pharmacists, which can help improve the quality of pharmaceutical care, and identify patients with migraines or analgesic abuse.

\section{REFERENCES AND/OR ACKNOWLEDGEMENTS}

Conflict of interest No conflict of interest 